\title{
How traumatic experiences leave their signature on the genome: an overview of epigenetic pathways in PTSD
}

\author{
Tania L. Roth* \\ Department of Psychological and Brain Sciences, University of Delaware, Newark, DE, USA \\ ${ }^{*}$ Correspondence: troth@psych.udel.edu \\ Edited and reviewed by: \\ Ming D. Li, University of Virginia, USA
}

Keywords: DNA methylation, histones, miRNA, stress, fear, PTSD

Epigenetic mechanisms are a class of molecular mechanisms by which environmental influences, including stress, can interact with the genome to have long-term consequences for brain plasticity and behavior. As PTSD, by definition, requires exposure to a traumatic event, and because genes are exquisitely sensitive to stress and trauma, epigenetic alterations have received attention as possible contributors to the development and persistence of PTSD symptoms. In this research topic, empirical support for the role of epigenetics in PTSD are presented and discussed. The articles assembled here cover a range of disciplines and experimental approaches in both animal models and humans that link stress and trauma with epigenetic alterations. Many articles also offer perspectives on epigenetics and sex differences, diagnosis and intervention, and future directions to bridge the gap between basic and clinical work.

Two original research articles provide empirical support for DNA methylation as a useful biomarker, not only in the detection and diagnosis of PTSD, but also as a biological measure for prediction of response to treatment, monitoring treatment efficacy, and prognosis of outcome. Rusiecki et al. (1) explore changes in DNA methylation of immune-related genes in US military service members with a PTSD diagnosis, showing differential patterns of methylation present pre- vs. post-deployment. Yehuda et al. (2) explore DNA methylation changes in association with changes in PTSD symptoms and other biological measures (including cortisol levels) in responders and non-responders to psychotherapy treatment.

Three review articles describe the compelling evidence for epigenetic alterations, particularly DNA methylation, as a consequence of exposure to stress encountered early in development. Raabe and Spengler (3) discuss studies showing early-life stress induced epigenetic alterations of stress genes as an important pathway in the dysregulation of stress systems in rodents and patients. Karsten and Baram (4) review the neuroanatomical and molecular pathways bridging sensory input with gene expression programing. They especially focus on how either nurturing or aversive early-life experiences can alter regulation of corticotropinreleasing hormone gene expression in hypothalamic neurons. McGowan (5) discusses studies of humans and animal model analogs that address molecular mechanisms underlying changes in stress-sensitive physiological systems in response to early-life trauma, paying particular attention to work on the glucocorticoid receptor. In an original research article, Kundakovic et al. (6) use a rodent model of early-life adversity (separation of infant mice from the mother) to explore the relationship between stress, genetic background, and sex in the determination of neurobehavioral and epigenetic outcomes. Together, data presented in these four articles are consistent with the notion that epigenetic programing early in life confers an enhanced risk on disease development upon re-exposure to trauma or stress. Throughout these articles, sex-specific differences at the epigenetic level are apparent too, suggesting that epigenetic activity plays an important role in sex-specificity and susceptibility to stress.

Continuing with a developmental theme, two review articles provide fascinating perspectives on the relationship between brain development and plasticity, gene $\times$ environment interactions, and the development of fear systems. Callaghan et al. (7) discuss developmental transitions in emotional learning and the role early-life stress has in both prematurely closing critical period plasticity and accelerating the development of fear learning systems. They also discuss the provocative idea of reopening critical periods of emotional learning to help treat many anxiety disorders. Nabel and Morishita (8) consider the potential contributions of "molecular brakes" identified in visual system development, the major model of critical period plasticity, to the development of fear system connections. They also discuss epigenetic regulators in the context of fear system development and their potential as new targets for therapeutic intervention.

One of the most common problems associated with PTSD is the persistence of memories of traumatic events. Since decades of research has shown that changes in gene expression occur when a memory is formed and stored, investigators have explored the relationship between DNA methylation and histone modifications and long-term trajectories in gene regulation associated with fear memories. These data are extensively described in two review articles. Maddox et al. (9) review the role of epigenetic mechanisms in animal models of fear learning and memory (Pavlovian fear conditioning paradigms that produce robust and long-lasting fear memories in rodents), highlighting epigenetic modulation of FKBP5 in animal models of PTSD and clinical populations. Zovkic et al. (10) review literature supporting the involvement of epigenetics in PTSD, discussing data in the broader context of epigenetics in stress and fear learning. They also focus on evidence for epigenetic mechanisms as regulators of predisposition and resilience to PTSD, and provide a technical overview of approaches for measuring DNA methylation to encourage 
future investigation of epigenetic mechanisms in animal models of PTSD.

Finally, though it is clear throughout this topic that DNA methylation has been the most extensively studied epigenetic alteration in outcomes associated with stress, evidence for histone modifications and microRNAs (miRNAs) as key epigenetic players are also emerging. Reul (11) discusses how psychologically stressful events evoke a long-term impact on behavior through changes in hippocampal function. Data are presented showing that this can occur through glutamatergic and glucocorticoid-driven changes in epigenetic regulation of gene transcription (via histone acetylation for example) within dentate gyrus neurons. In an original research article, Schmidt et al. (12) explore cortical miRNA expression profiles in a rodent model of PTSD. miRNAs are a more recent recognized class of epigenetic modulators of gene activity (or even a regulator of epigenetic processes), and are small non-coding RNAs that can regulate gene expression post-transcriptionally. Selective serotonin reuptake inhibitors (SSRIs) are the only FDA approved treatment for PTSD, with some evidence that one SSRI, fluoxetine, can ameliorate a subset of PTSD symptoms. These authors also examine fluoxetine effects on miRNA profiles, which may provide insight into the mechanisms underlying treatment effects of antidepressants in PTSD.

\section{ACKNOWLEDGMENTS}

I thank co-editors David Diamond and Karestan Koenen for comments, and work on this editorial was supported by a grant from The National Institute of General Medical Sciences (1P20GM103653).

\section{REFERENCES}

1. Rusiecki JA, Byrne C, Galdzicki Z, Srikantan V, Chen L, Poulin M, et al. PTSD and DNA methylation in select immune function gene promoter regions: a repeated measures case-control study of U.S. military service members. Front Psychiatry (2013) 4:56. doi:10.3389/fpsyt.2013.00056

2. Yehuda R, Daskalakis NP, Desarnaud F, Makotkine I, Lehrner A, Koch E, et al. Epigenetic biomarkers as predictors and correlates of symptom improvement following psychotherapy in combat veterans with PTSD. Front Psychiatry (2013) 4:118. doi:10.3389/fpsyt.2013.00118

3. Raabe FJ, Spengler D. Epigenetic risk factors in PTSD and depression. Front Psychiatry (2013) 4:80. doi:10.3389/fpsyt.2013.00080
4. Karsten CA, Baram TZ. How does a neuron "know" to modulate its epigenetic machinery in response to early-life environment/experience? Front Psychiatry (2013) 4:89. doi:10.3389/fpsyt.2013.00089

5. McGowan PO. Epigenomic mechanisms of early adversity and HPA dysfunction: considerations for PTSD research. Front Psychiatry (2013) 4:110. doi:10.3389/fpsyt.2013.00110

6. Kundakovic M, Lim S, Gudsnuk K, Champagne FA. Sex-specific and straindependent effects of early life adversity on behavioral and epigenetic outcomes. Front Psychiatry (2013) 4:78. doi:10.3389/fpsyt.2013.00078

7. Callaghan BL, Graham B, Li S, Richardson R. From resilience to vulnerability: mechanistic insights into the effects of stress on transitions in critical period plasticity. Front Psychiatry (2013) 4:90. doi:10.3389/fpsyt.2013.00090

8. Nabel EM, Morishita H. Regulating critical period plasticity: insight from the visual system to fear circuitry for therapeutic interventions. Front Psychiatry (2013) 4:doi:10.3389/fpsyt.2013.00146

9. Maddox SA, Schafe GE, Ressler KJ. Exploring epigenetic regulation of fear memory and biomarkers associated with post-traumatic stress disorder. Front Psychiatry (2013) 4:62. doi:10.3389/fpsyt.2013.00062

10. Zovkic I, Meadows JP, Kaas GA, Sweatt JD. Interindividual variability in stress susceptibility: a role for epigenetic mechanisms in PTSD. Front Psychiatry (2013) 4:60. doi:10.3389/fpsyt.2013.00060

11. Reul JM. Making memories of stressful events: a journey along epigenetic, gene transcription and signaling pathways. Front Psychiatry (2014) 5:5. doi:10.3389/ fpsyt.2014.00005

12. Schmidt U, Herrmann L, Hagl K, Novak B, Huber C, Holsboer F, et al. Therapeutic action of fluoxetine is associated with a reduction in prefrontal cortical miR-1971 expression levels in a mouse model of posttraumatic stress disorder (PTSD). Front Psychiatry (2013) 4:66. doi:10.3389/fpsyt.2013. 00066

Conflict of Interest Statement: The author declares that the research was conducted in the absence of any commercial or financial relationships that could be construed as a potential conflict of interest.

Received: 14 July 2014; accepted: 16 July 2014; published online: 31 July 2014. Citation: Roth TL (2014) How traumatic experiences leave their signature on the genome: an overview of epigenetic pathways in PTSD. Front. Psychiatry 5:93. doi: 10.3389/fpsyt.2014.00093

This article was submitted to Molecular Psychiatry, a section of the journal Frontiers in Psychiatry.

Copyright $(9) 2014$ Roth. This is an open-access article distributed under the terms of the Creative Commons Attribution License (CC BY). The use, distribution or reproduction in other forums is permitted, provided the original author(s) or licensor are credited and that the original publication in this journal is cited, in accordance with accepted academic practice. No use, distribution or reproduction is permitted which does not comply with these terms. 